\title{
Nutraceuticals in Arthritis Management: A Contemporary Prospect of Dietary Phytochemicals
}

\author{
Md. Nur Alam, Mohammad Mijanur Rahman and Md. Ibrahim Khalil*
}

Department of Biochemistry and Molecular Biology, Jahangirnagar University, Savar, Dhaka-1342, Bangladesh.

\begin{abstract}
Rheumatoid Arthritis is the most common, chronic autoimmune disease with no known cure, and a priority for biological research in the twenty first century. The limitations in the efficacy and safety of current treatment strategies are driving the need for new, safe and effective medications. Recent experimental findings focus on the potential of neutraceuticals for the management of rheumatoid arthritis. In this review, we summarize the effect of several dietary phytochemicals against RA with important biochemical findings. A number of dietary phytochemicals can either retard or improve the RA by their anti-oxidative, anti-inflammatory, immunomodulatory and enzyme inhibitory effects. This review suggests that the dietary phytochemicals have immense potential, to be developed as safe and effective treatment options. More research is required to understand how dietary phytochemicals affect rheumatoid arthritis.
\end{abstract}

Keywords: Dietary phytochemicals, neutraceutical, rheumatoid arthritis.

\section{INTRODUCTION}

Arthritis is a chronic disease affecting the musculoskeletal system, particularly the joints. Generally, arthritis is characterized by pain, swelling and stiffness of one or more joints due to inflammation, irrespective of the underlying causes. There are over 100 types of arthritis, each with defined medical presentations, prevalence and prognoses. Osteoarthritis, rheumatoid arthritis, septic arthritis and psoriatic arthritis are the most common forms of arthritis. Rheumatoid Arthritis (RA) is the most common autoimmune disease and the second most familiar arthritis compared to osteoarthritis [1, 2]. It is characterized by an autoimmune response that causes chronic inflammatory destruction of synovial joints $[1,2]$. RA is associated with progressive disability, systemic complications and premature death [3]. Specifically, uncontrolled active RA causes joint damage, disability, decreased quality of life, and cardiovascular and other comorbidities [4]. RA affects approximately $1 \%$ of adults all over the world with 2-3 times higher occurrence in women than men [5]. The prevalence of RA generally rises with increasing age until approximately 70 years old. The risk of getting RA in developing countries generally peaks at earlier ages, with the peak age varying by particular location $[6,7]$.

Standard treatment recommends a combination of nonpharmacologic and pharmacologic therapies. The pharmacological treatment of RA usually aims to prevent further progression of the disease by relieving symptoms using disease-modifying anti-rheumatic drugs (DMARDs) $[8,6]$. Non-pharmacologic therapies include lifestyle changes,

*Address correspondence to this author at the Department of Biochemistry and Molecular Biology, Jahangirnagar University, Savar, Dhaka-1342, Bangladesh; Tel: +88 01711 034983; Fax: +88 02 7708069;

E-mail:drmikhalil@yahoo.com which vary depending on the patient's level of disability, intended to improve their quality of life [9]. Reduced progression of the disease and improved quality of life can be achieved through the use of corticosteroids and biological agents in the early course of the disease [10]. There are significant side effects from these treatments in the later stages of the disease, which affect the overall benefit of using corticosteroids and biological agents. The trade-off between the early benefit of these interventions and the later negative consequences need to be considered in planning the treatment of each patient.

A plethora of evidence suggests that diet and nutrient supplementation has the potential for improving RA management. These potential benefits are achieved by alleviating symptoms, and slowing the progression of the disease or by reducing the reliance on, or combating the sideeffects of pharmacologic therapy [11, 12]. Historically, patients diagnosed with RA have tried changing their diets in the hope that it may improve their symptoms. Patients frequently sought dietary advice, in an attempt to include nutritional changes as an additional treatment modality. The patients were reluctant to use pharmacological treatment exclusively for treating RA because of the negative side effects $[13,14]$. The therapeutic efficacy achieved through diet is based on one or a set of phytochemicals, most often the physiologically active secondary metabolites like polyphenol, flavonoid, tannin, anthocyanine, glycoside etc. The manipulation of diet is widely used by patients worldwide in the recent time, often involving the inclusion and/or exclusion of food items. Systematic scientific consideration and analyses of the effect of these dietary changes has not been undertaken and there is little understanding of the effect of inclusion or exclusion of particular phytochemicals in the diet and how they function in the body to result in particular outcomes [15]. Therefore, 
we are interested in reviewing the therapeutic value of dietary phytochemicals in a mechanistic view.

In the present review, we first go through the pathophysiological basis of RA and identify the therapeutic focus. Second, we will focus on the effect of dietary phytochemicals, in particular the effect of polyphenols and flavonoids on the prevention of the progression of RA. Finally, we will suggest several promising future prospects of dietary phytochemicals in RA.

\section{PATHOGENESIS OF RHEUMATOID ARTHRITIS}

Physiologically, rheumatoid arthritis is characterized by synovial inflammation and hyperplasia, autoantibody production, cartilage and bone destruction [16]. The molecular pathogenesis suggests a complex interplay between the genetic and environmental factors leading to an abstruse development of the autoimmune response as the underlying event triggering the RA [4]. Large genome-wide association studies have identified more than 30 loci involved in RA pathogenesis. Both, HLA (e. g. DRB1*01, DRB1*04, DRB1*13 and DRB1*15) and some non-HLA (e. g. PTPN22, IL23R, TRAF1, CTLA4, IRF5, STAT4, CCR6, PADI4.) genes are known to predispose to RA heritability with greater contribution by HLA genes toward RA, while genetic susceptibility has also been associated with environmental factors Fig. (1) [17].

The development of an autoimmune response is the central tounderstanding RA, and is based onan intricate gene-environment interaction leading to the loss of immune tolerance and consequent microenvironmental changes of the synovial [16]. An unbalanced immune system provides the molecular stimulation, through complex cell-cell interactions (involving local dendritic cells that express cytokines, HLA class II molecules, and co-stimulatory molecules) necessary for T-cell activation and proliferation [18, 19]. T-cell activation causes antigen presentation and ' $\mathrm{T}$-cell-mediated B-cell help' that results in activation of $B$ cells and macrophages [20]. Macrophages are the central effectors of synovitisact through the release of cytokines (e.g., TNF- $\alpha$ and interleukin-1, 6, 12,15,18,23), production of prostanoids and matrix-degrading enzymes, phagocytosis and antigen presentation [21]. The proinflammatory cytokines like interleukin-1 and tumor necrosis factor- $\alpha$ (TNF $\alpha)$ that

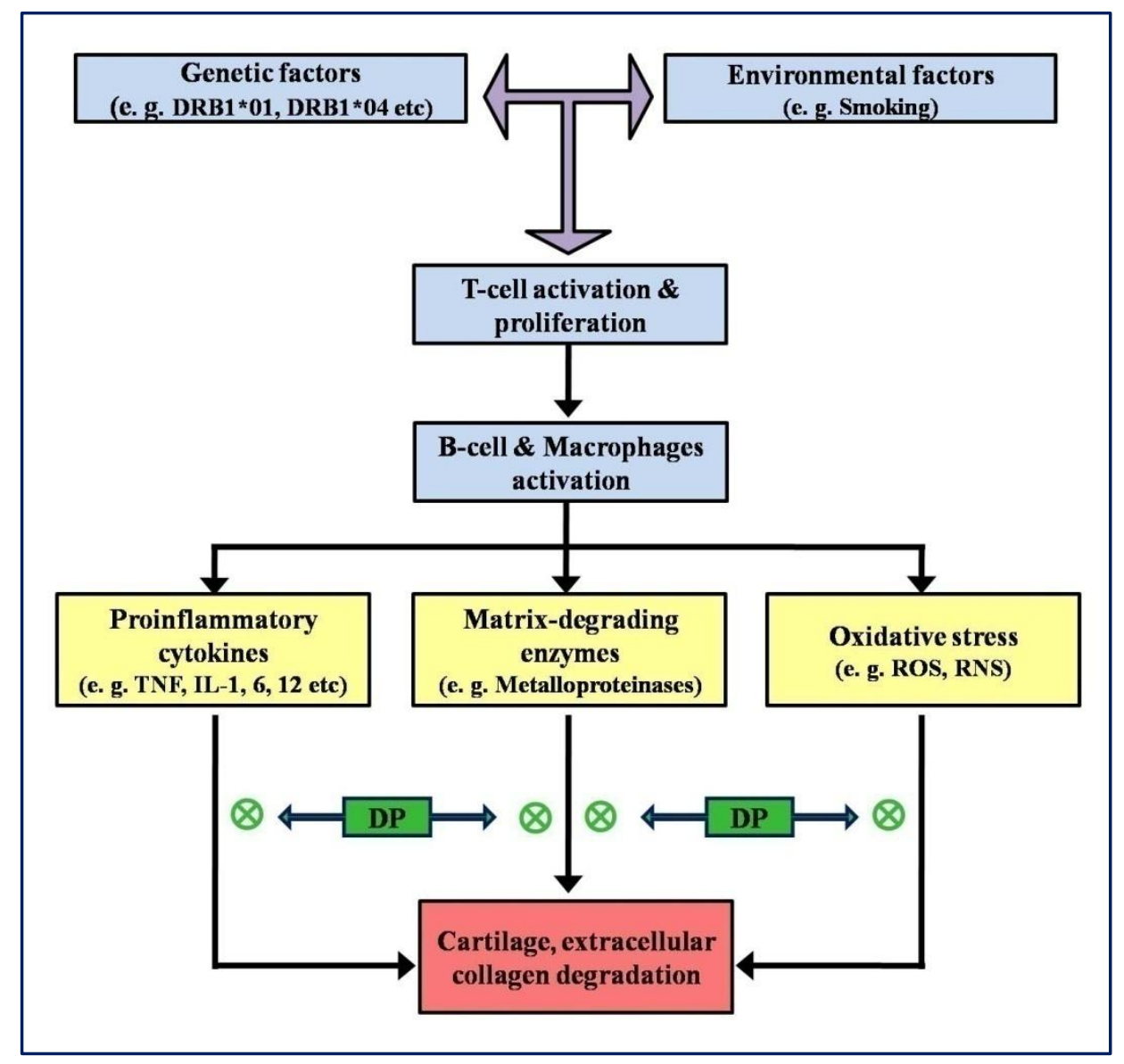

Fig. (1). Overview of RA pathophysiology. Here, ROS = Reactive oxygen species;RNS = reactive nitrogen species; DP = Dietary phytochemicals. Intricate interaction between an array of genetic and environmental factors predispose to overwhelmed immune response in an autoimmune fashion that involve pathogenic activation of T-lymphocytes, B-lymphocytes and macrophages. Such pathogenic immune activation causes oxidative stress, inflammation and activation of matrix degrading enzymes those ultimately leads to manifestation of RA like cartilage degradation, extracellular collagen degradation etc. Besides antioxidative effect, several dietary phytochemicals like resveratrol,firulic acid, curcumin, genisteinetc halt or ameliorate inflammation. Several dietary phytochemicals like firulic acid, gallic acid etc also inhibit the activation of degrading enzyme to restore the cartilage structure. 
stimulate synovial fibroblasts and chondrocytes in articular cartilage to secrete enzymes that degrade proteoglycans and collagen, leading to tissue destruction [22]. Beside cytokines reactive oxygen intermediates and nitrogen intermediates leads to oxidative stress is significantly involved in degradation of cartilage, extracellular collagen and intracellular DNA while antioxidants and oxidative enzymes have been shown to ameliorate arthritis [23].

\section{DIETARY PHYTOCHEMICALS IN RHEUMATOID ARTHRITIS}

The human diet contains a large range of food items of plant and animal origin. Food of plant origin covers a large range of phytochemicals including polyphenols, flavonoid, alkaloid, glycosides, terpenoids, phytosterols sterols etc. The contributions of such phytochemical constituents on rheumatoid arthritis have been described below in brief.

\section{Apigenin}

Apigenin is a dietary flavonoid of flavone class found in fruits, vegetables, spices and herbs. Activated RA fibroblastlike synoviocytes play a central role in both initiating and driving RA. Apigenin inhibited the collagenase activity involved in RA [24]. Apigenin enhances the cytotoxic effects of TNF-related apoptosis-inducing ligand in human rheumatoid arthritis fibroblast-like synoviocytes which is likely mediated through inactivation of PI3-K/Akt signaling pathway [25]. Shin et al. also suggested that the apigenininduced apoptosis of rheumatoid fibroblast-like synoviocytes is mediated by reactive oxygen species and activation of ERK1/2 [26]. Several other studies have already been performed to clarify the related anti-inflammatory mechanism of apigenin, leading to hopeful results. Apigenin suppressed lipopolysaccharide (LPS)-induced nitric oxide (NO) production in a dose dependent manner in macrophage cells (RAW 264.7) with the attenuation of cyclooxygenase-2 (COX-2) expression. Pretreatment with apigenin also reduced TNFa-induced adhesion of monocytes and expression of cellular adhesion molecules upregulation of vascular cellular adhesion molecule-1 (VCAM-1), intracellular adhesion molecule-1 (ICAM-1), and E-selectin. Apigenin inhibits the production of pro-inflammatory cytokines IL-1 $\beta$, IL-8, and TNF in LPS-stimulated human monocytes and mouse macrophages [27]. At the molecular level such anti-inflammatory activity of Apigenin is mediated by the suppression of NF-kB activation while nuclear translocation and DNA binding activity of NF- $\mathrm{KB}$, p65 and the inactivation of the IKK complex were concomitantly evident [28]. Such a profound antiinflammatory strategy obviously encourages the therapeutic use of apigenin in the chronic inflammatory situation like RA.

\section{Arctigenin}

Arctigenin is a naturally occurring phenylpropanoiddibenzylbutyrolactone lignin. There exist no direct clues to the anti-RA effect of arctigenin but several promising findings suggest the probable anti-RA effect of arctigenin. The study of Zhao et al. provided an initial clue of the antiinflammatory mechanism of arctigenin. Their study showed that arctigenin suppressed lipopolysaccharide (LPS)stimulated NO production through down-regulation of iNOS expression and iNOS enzymatic activity and proinflammatory cytokines secretion, including TNF-alpha and IL-6 in a dose-dependent manner in LPS-stimulated macrophage [29]. An another degree of resolution of antiinflammatory mechanism of arctigenin was from the studies of Hyam et al. with the finding that arctigenin inhibited NF$\kappa \mathrm{B}$ activation, as well as PI3K, AKT and IKK $\beta$ phosphorylation in association with the inhibition of IL-1 $\beta$, TNF- $\alpha$ and IL- 6 expression under inflammatory situation in vivo [30]. Arctigenin suppresses the activation of MAP kinases (ERK1/2, p38 kinase and JNK) through the inhibition of MKK activities, resulting to AP-1 inactivation that might inhibit the TNF- $\alpha$ production [31]. The arctigenin also exerted its anti-inflammatory effect by inhibiting ROSdependent STAT signaling through its antioxidant activity [32]. Another study also indicates passive evidence of the antioxidant activity of the arctigenin that support antioxidant activity mediated anti-inflammatory activity of arctigenin [33]. Thus, arctigenin may be used in the RA associated inflammatory complications.

\section{Curcumin}

Curcumin is the major curcuminoid of turmeric (Curcuma longa). Intraperitoneal injection of extract containing curcuminoids prior to arthritis induction significantly inhibited acute (75\%) and chronic (68\%) inflammation of joint [34]. Chandran \& Goel first suggested the safety and superiority of curcumin treatment in patients with active RA [35]. Curcumin has reported to mediate antiinflammatory effects through the down regulation of inflammatory enzymes like nitric oxide synthase (iNOS), COX 2 and Arachidonate5-lipoxygenase (ALOX5); various pro-inflammatory cytokine expressions like tumor necrosis factor (TNF- $\alpha$ ), interleukins (IL-1, IL-2, IL-6, IL-8, IL-12) and chemokines. All these anti-inflammatory strategies of curcumin are mediated through inactivation of the nuclear transcription factor nuclear factor kappa B (NF- $\kappa B$ ) while $\mathrm{NF}-\kappa \mathrm{B}$ is the molecular correspondence of inflammatory responses in arthritic patient [36-38]. Current report suggests that curcumin is an alternative TNF- $\alpha$ blocker in vitro and in vivo. Interestingly, low dose of curcumin is clinically effective in the management and treatment of osteoarthritis [11]. Moreover, curcumin is an efficient scavenger of free radicals while free radical damage underlies chronic inflammation [39, 40]. Curcumin showed superb intracellular ROS scavenging activity where it easily transfer electron or donate $\mathrm{H}$-atom from two phenolic sites to scavenge free radicals [40]. However, the process of arthritis specific anti-inflammatory reactions seen when using curcumin needs to be understood in more detail.

\section{Epigallocatechin-3-Gallate (EGCG)}

Epigallocatechin-3-gallate (EGCG) is the ester of epigallocatechin and gallic acid and the major active component of green tea (Camellia sinensis). EGCG has significant inhibitory effect on the collagen and Interleukin-1 beta (IL-1 $1 \beta$ ) - induced inflammatory responses. The antiarthritis activity of EGCG was first discovered from the 
finding that consumption of EGCG-containing green tea ameliorated collagen-induced arthritis in mice. This improvement in arthritis was associated within a marked inhibition of the inflammatory mediators cyclooxygenase 2 (COX-2), Interferon gamma (IFN $\gamma$ ), and tumor necrosis factor alpha $(\mathrm{TNF} \alpha)$ in arthritic joints, lower level of total immunoglobulins ( $\operatorname{IgG}$ ) and type II collagen-specific $\operatorname{IgG}$ in serum and arthritic joints [41]. Pre-incubation of human chondrocytes derived from arthritic cartilage with EGCG inhibits the IL-1 $\beta$-induced inducible nitric oxide synthase (iNOS) and COX-2 expression and activity that ultimately resulted in the reduced nitric oxide (NO) and prostaglandin E2 synthesis (PGE2) [42, 43]. The study of Lin et al. also provided a mechanistic clue to EGCG's effect on arthritis. They found that intraperitoneal administration of EGCG ameliorated arthritis and macrophage infiltration, and caused a reduction in the amount of MCP-1/CCL2-synthesizing osteoblasts [44]. The anti-inflammatory and anti-arthritic effects of EGCG are also supported by in vitro and in vivo data indicating that EGCG or EGCG containing green tea can regulate the expression of cytokines, chemokines, MMPs, aggrecanase, ROS, NO, COX-2, and PGE2 in cell type relevant to the pathogenesis of OA and RA [45]. Besides these, EGCG has been reported to have bonepreserving and synovial fibroblast regulatory effect [46]. EGCG is also a potent antioxidant with mounting evidences. Epigallocatechingallate effectively scavenge superoxide anion, hydroxyl radical and other reactive oxygen species (ROS) while ROS has a pathogenic role in rheumatoid arthritis and excessive quantities of oxygen free radicals have been identified in synovial fluid of $90 \%$ of patients with RA [47, 48]. Several studies have shown that EGCG blunts ROS mediated cytotoxicity in human chondrocytes and other models of oxidative stress while considerably increased the gene expressions of catalase, superoxide dismutase, and glutathione peroxidase activities which are essential components of a robust anti-oxidant defense system [49]. Therefore, EGCG may be a potential therapeutic agent against arthritis offering incredible new options in the treatment of arthritis as well as better quality of life for arthritic patients.

\section{Ferulic Acid}

Ferulic acid is a natural organic compound of polyphenol class. Available evidence suggests that ferulic acid counteracts arthritis associated inflammation in different trials either remarkably or partly. Phenolic compounds rich fraction containing ferulic acid significantly inhibited the adjuvant-induced arthritis while dimerization of the ferulic acid has been reported to inhibit lipopolysaccharide (LPS)induced COX-2 expression in the mouse macrophage [50]. Co-incubation of sodium ferulate (a sodium salt of ferulic acid) with chondrocytes while IL-1 $\beta$ causes a significant attenuation of apoptosis rate of chondrocytes. Such an amelioration was concurrent with the decreased expressions of TNF $\alpha$, TNFR-1, TNF receptor-associated death domain, caspase- 8 and caspase- 3 in a dose-dependent manner [51]. In vivo experiments, it was revealed that ferulic acid pretreatment lowers the level of hydrogen peroxide-induced IL$1 \beta$, TNF- $\alpha$, and MMP-1 whereas decreased expression of MMP-1 and MMP-13 during ferulic acid post-treatment
[41]. Sodium ferulate (SF), a natural derivative of ferulic acid, significantly ameliorated acetic acid induced inflammatory responses in rats, with the lowered myeloperoxidase (MPO) activity, superoxide dismutase (SOD) activity, malondialdehyde (MDA) levels, nitric oxide (NO) levels, PGE2 levels. SF reduces the expressions of iNOS, COX-2 and nuclear factor kappa B (NF-kB) p65 proteins in the colonic tissue [52]. SF also reverses cartilage degradation processes and inhibits expressions of matrix metalloproteinase-1 and BAX in a rat OA model. SF prevents apoptosis of chondrocytes, represses NO synthesis and attenuates the levels of matrix metalloproteinase-1/tissue inhibitor of metalloproteinase-1 to prevent extracellular matrix degradation in human OA chondrocytes [53]. The study of Roy et al. revealed a significant reduction in the activity of apoptosis and reduced expression of TGF- $\beta 1$ and IL-1 $\beta$ in the pancreatic $\beta$-cell due to ferulic acid treatment in rats [54]. Moreover, FA may contribute to the prevention of arthritis with the antioxidant and radical scavenging potential in a passive way.

\section{Gallic Acid}

Gallic acid (3, 4, 5-trihydroxybenzoic acid), a natural polyphenolic acid found in gall nuts, sumac, oak bark, tea leaves, grapes and win. Several alternative finding suggest to figure out the anti-RA effect of gallic acid. Gallic acid treatment inhibited the expression of several proinflammatory genes in dose and time dependent manner in TNF-a treated fibroblast like synoviocyte derived from patients with rheumatoid arthritis (RA FLS) [55]. Gallic acid also induces caspase-3 dependent apoptosis of RA FLS [56]. Furthermore, gallic acid possesses a profound antiinflammatory effect [57]. Gallic acid inhibits histamine release and pro-inflammatory cytokine TNF- $\alpha$ and IL-6 production in human mast cells [58]. Therefore, gallic acid has immense potential to treat inflammatory condition while RA is a chronic inflammatory situation.

\section{Genistein}

Genistein is a phytoestrogen of isoflavone class. Genistein inhibits the growth of rheumatoid arthritis fibroblast-like synoviocytes under the stimulation of cytokines (IL-1 $\beta$ \& TNF- $\alpha$ ) and growth factor-induced (EGF). Such an inhibition was marked by decreased cell number, tritiated thymidine incorporation and cell cycle arrest at the G1 restriction point; genistein decreased colony numbers under anchorage-independent condition stimulated by EGF in serum [59]. In the collagen-induced rheumatoid arthritis rat model, genistein modulated a Th1-predominant immune response with significant suppression of the secretion of IFN- $\gamma$, and it augmented the IL-4 production thus, keeping the Th1/Th2 balance [60]. In macrophage cell line (RAW 264.7), genistein effectively inhibited the LPSinduced overproduction of TNF- $\alpha$, IL- 6 , and NF- $\mathrm{KB}$ activation [61]. In inflammation, genistein inhibited iNOS protein and NO production in a dose-dependent manner concomitantly with the inhibition of STAT-1 and NFkappaB activations [62]. Genistein also significantly suppressed TNF- $\alpha$-induced production of adhesion molecules and chemokines such as sICAM-1, sVCAM-1, 
sE-Selectin, MCP-1 and IL-8, which play key role in the firm adhesion of monocytes to activated endothelial cells (ECs) [63].

\section{Kaempferol}

Kaempferol is a natural flavonol of the flavonoid type. Kaempferol has the capacity to inhibit the proliferation of both normal and IL-1 $\beta$ stimulated rheumatoid arthritis synovial fibroblasts (RASFs), which are involved in the destruction of both articular bone and cartilage. This inhibitory activity of Kaempferol was marked by the down expression of MMP-1, MMP-3, COX-2 and PGE2 induced by IL-1 $\beta$. Kaempferol, which also inhibited the phosphorylation of ERK-1/2, p38 and JNK, the activation of NF- $\kappa B$ induced by IL-1 $\beta$ as well [64]. In LPS-activated macrophage, kaempferol inhibited iNOS expression and NO production in a dose-dependent manner. In addition, Kaempferol inhibited the activation of the STAT-1(Signal transducer and activator of transcription 1) that is another important transcription factor for iNOS expression [62]. The study of Gong et al. also provides a mechanistic clue to the anti-inflammatory activity of kaempferol. They found that kaempferol can suppress eosionphil infiltration as well as a dose-dependent attenuation of TNF $\alpha$-induced expression of epithelial intracellular cell adhesion molecule-1 and eosinophil integrin $\beta 2$ that contribute to the amelioration of allergic and inflammatory airway diseases [65]. Kaempferol inhibits IL-4-induced STAT6 activation while IL-4-driven STAT6 activation has been reported to play an active role in the progression of inflammatory diseases [66]. All these adumbrate an implicit therapeutic potential of kaempferol against RA.

\section{Quercetin}

Quercetin is a flavonoid. In rheumatoid arthritis fibroblast-like synoviocytes, quercetin causes apoptosis in a dose-dependent manner concomitantly with enhanced caspase-3 and caspase-9 cleavages [67]. Quercetin caused a concentration-dependent loss of mitochondrial membrane potential and cytochrome $\mathrm{c}$ release to cytosol and also decreased $\mathrm{Bcl}-2 / \mathrm{Bax}$ ratio, indicating that quercetin-induced apoptosis is through mitochondrial pathway [68]. In chronic rat adjuvant-induced arthritis, quercetin inhibits macrophagederived cytokines and nitric oxide (NO) and thus possess potential anti-inflammatory therapeutic and preventive agent targeting the inflammatory response of macrophages [69]. Quercetin supplementation reduced tumor necrosis factoralpha and interleukin-1beta mRNA levels simultaneously with reduced levels of circulating macrophage chemoattractant protein 1 , nitric oxide, and prostaglandin $E(2)$ [70]. In another study, quercetin inhibited neutrophil activation, synoviocyte proliferation, and angiogenesis [71].

\section{Resveratrol}

Resveratrol is a polyphenol present at high concentration in grape skin, seeds, and red wine. Resveratrol prevents synovial hyperplasia by inducing the by caspase- 9 dependant apoptosis of human rheumatoid arthritis synovial cells (MH7A) [72]. Byun et al. (2008) showed that the resveratrol induces the apoptosis of fibroblast-like synoviocytes derived from patients with rheumatoid arthritis by activating caspase- 8 and caspase- 9 without affecting the levels of Bax, Bcl-XL, and Bcl-2 [73]. Several other researchers also provide a mechanistic view toward the anti-RA effect of resveratrol. Nuclear factor kappa B (NF- $\kappa B)$ is a pivotal transcription factor in synovial cells, and is involved in the activation of the TNF- $\alpha$ and IL-1 $\beta$ genes while resveratrol inhibits TNF- $\alpha$ and IL-1 $\beta$ expression suppressing NF- $\mathrm{KB}$ activity in human chondrocytes [74]. The chondro-protective effects of resveratrol were associated with suppression of iNOS and COX-2 [75]. Resveratrol modulates collageninduced arthritis by inhibiting Th17 and B-cell function that lies at upstream point in RA pathogenesis [76]. The antioxidant property of resveratrol can also be involved in the anti-RA effect as the resveratrol reduce NO levels resulting reduced physiological oxidative stress [77]. In addition, resveratrol was found to be an effective scavenger of hydroxyl, superoxide, and metal-induced radicals as well as showing antioxidant abilities in cells producing ROS [78]. Therefore, the pharmacological property of resveratrol might be used against RA associated inflammatory responses.

\section{CONCLUDING REMARK}

RA is a chronic disease with no known cure that affects many people. It is an important challenge for biological research in the twenty first century. Despite of supportive treatment, current treatment medications are limited in their efficacy and are frequently toxic. With the growing interest in neutraceuticals among people with RA, patients often look for dietary therapeutic options to treat their condition. Patient's need a more thorough and systematic investigation of the effect of dietary phytochemicals on RA to educate their treatment decisions.

The wide and varying range of symptoms involved in RA, such as inflammation, oxidative stress and other secondary events have made the RA an illness that is difficult to diagnose and treat. This review clearly suggests that dietary photochemicals have immense potential, to be developed as a safe and effective treatment option while targeting RA. A number of dietary phytochemicals can retard or improve the RA symptoms through their anti-oxidative, anti-inflammatory, immunomodulatory and enzyme inhibitory effects.

More research should be designed to evaluate the influence of different dietary phytochemicals in RA specific situations. There is a large and important opportunity to figure out the precise effect of dietary phytochemicals in upstream pathophysiological points of RA like T-lymphocyte activation, B-lymphocyte activation, phagocytes activation etc. The effect of dietary phytochemicals in proinflammatory response, oxidative stress associated with RA and other enzyme system especially of the degrading type may elicit excellent supportive treatment approaches. In that respect, utilizing both cellular and animal models may help to reveal the extent of the medicinal influence. Finally, clinical studies using human subjects should be used to test the potential, safety and efficiency of dietary phytochemicals. 


\section{CONFLICT OF INTEREST}

The authors confirm that this article content has no conflicts of interest.

\section{ACKNOWLEDAMENTS}

Decleared none.

\section{REFERENCES}

[1] O'Dell JR. Rheumatoid arthritis. $24^{\text {th }}$ ed. In: Goldman L, Schafer AI, Ed. Goldman's Cecil Medicine. Philadelphia, PA: Saunders Elsevier, 2011: pp. 1681-9.

[2] Firestein GS. Etiology and pathogenesis of rheumatoid arthritis. 8 ed. In: Firestein GS, Budd RC, Harris ED Jr, et al. Ed. Kelley's Textbook of Rheumatology. Philadelphia, PA: Saunders Elsevier, 2008: pp. 1035-86.

[3] Ramos M, Tak P, Lebre M. Fms-like tyrosine kinase 3 liganddependent dendritic cells in autoimmune inflammation. Autoimmun Rev 2014; 13(2): pp. 117-24.

[4] Scott DL, Frederick WF, Huizinga TWJ. Rheumatoid arthritis. Lancet 2010; 376(97460): 1094-08.

[5] Clements JN. Treatment of rheumatoid arthritis: a review of recommendations and emerging therapy. Formulary 2011; 46: 53245 .

[6] Symmons D, Mathers C, Pfleger B. The global burden of rheumatoid arthritis in the year 2000 [monograph on the internet]. Geneva: WHO; 2006. Available from: http://www.who.int/ healthinfo/statistics/bod_rheumatoidarthritis.pdf

[7] Costenbader K, Chang S, Laden F, Puett R, Karlson EW. Geographic variation in rheumatoid arthritis incidence among women in the United States. Arch Intern Med 2008; 168: 1664-70.

[8] Donahue K, Gartlehner G, Jonas D, et al. Systematic review: comparative effectiveness and harms of disease-modifying medications for rheumatoid arthritis. Ann Intern Med 2008; 148: 124-34.

[9] Christie A, Jamtvedt G, Dahm K, Moe R, Haavardsholm EA, Hagen KB. Effectiveness of nonpharmacological and nonsurgical interventions for patients with rheumatoid arthritis: an overview of systematic reviews. Phys Ther 2007; 87: 1697-715.

[10] Klarenbeek NB, Kerstens PJSM, Huizinga TWJ, Dijkmans BA, Allaart CA. Recent advances in the management of rheumatoid arthritis. BMJ 2010; 341 doi: http://dx.doi.org/10.1136/bmj.c6942 (Published 21 December 2010) ; Cite this as: BMJ 2010;341:c6942

[11] Rennie K, Hughes J, Lang R, Jebb S. Nutritional management of rheumatoid arthritis: a review of the evidence. J Hum Nutr Dietet 2003; 16: 97-109.

[12] Kjeldsen-Kragh J, Haugen M, Borchgrevink C, Førre O. Vegetarian diet for patients with rheumatoid arthritis--status: two years after introduction of the diet. Clin Rheumatol 1994; 13(3): 475-82.

[13] Smedslund G, Byfuglien M, Olsen S, Hagen K. Effectiveness and safety of dietary interventions for rheumatoid arthritis: a systematic review of randomized controlled trials. J Am Diet Assoc 2010; 110(5): 727-35.

[14] Muller H, de Toledo F, Resch K. Fasting followed by vegetarian diet in patients with rheumatoid arthritis: a systematic review. Scand J Rheumatol 2001; 30(1): 1-10.

[15] Vitetta L, Coulson S, Schloss J, Beck S, Allen R, Sali A. Dietary recommendations for patients with rheumatoid arthritis: a review. Nutr Diet Suppl 2012; 4: 1-15.

[16] McInnes IB, Schett G. The Pathogenesis of Rheumatoid Arthritis. J Med 2011; 365: 2205-19.

[17] Kurkó J, Besenyei T, Laki J, Glant TT, Mikecz K, Szekanecz Z. Genetics of Rheumatoid Arthritis - a Comprehensive Review. Clin Rev Allergy Immunol 2013; 45(2): 170-9.

[18] Lebre MC, Jongbloed SL, Tas SW, Smeets TJ, McInnes IB, Tak PP. Rheumatoid arthritis synovium contains two subsets of CD83DC-LAMP- dendritic cells with distinct cytokine profiles. Am J Pathol 2008; 172: 940-50.

[19] Schroder AE, Greiner A, Seyfert C, Berek C. Differentiation of B cells in the nonlymphoid tissue of the synovial membrane of patients with rheumatoid arthritis. Proc Natl Acad Sci USA 1996; 93: $221-5$
[20] Cantaert T, Brouard S, Thurlings RM, et al. Alterations of the synovial $\mathrm{T}$ cell repertoire in anti-citrullinated protein antibodypositive rheumatoid arthritis. Arthritis Rheum 2009; 60: 1944-56.

[21] Cutolo M, Kitas GD, Riel, PL. Burden of disease in treated rheumatoid arthritis patients: Going beyond the joint. Semin Arthritis Rheum 2014; 43(4): 479-88.

[22] Arend W. The pathophysiology and treatment of rheumatoid arthritis. Arthritis Rheum 1997; 40(4): 595-7.

[23] Hitchon CA, El-Gabalawy HS. Oxidation in rheumatoid arthritis. Arthritis Res Ther 2004; 6: 265-78.

[24] Lee JH, Zhou HY, Cho SY, Kim YS, Lee YS, Jeong CS. Antiinflammatory mechanisms of apigenin: inhibition of cyclooxygenase- 2 expression, adhesion of monocytes to human umbilical vein endothelial cells, and expression of cellular adhesion molecules. Arch Pharm Res 2007; 30(10): 1318-27.

[25] Sun QW, Jiang SM, Yang K, Zheng JM, Zhang L, Xu WD. Apigenin enhances the cytotoxic effects of tumor necrosis factorrelated apoptosis-inducing ligand in human rheumatoid arthritis fibroblast-like synoviocytes. Mol Biol Rep 2012; 39(5): 5529-35.

[26] Shin GC, Kim C, Lee JM, et al. Apigenin-induced apoptosis is mediated by reactive oxygen species and activation of ERK1/2 in rheumatoid fibroblast-like synoviocytes. Chem Biol Interact 2009; 182(1): 29-36.

[27] Nicholas C, Batra S, Melissa A, et al. Apigenin Blocks Lipopolysaccharide-Induced Lethality In Vivo and Proinflammatory Cytokines Expression by Inactivating NF- $\mathrm{KB}$ through the Suppression of p65 Phosphorylation. J Immunol 2007; 179(10): 7121-7.

[28] Funakoshi-Tago M, Nakamura K, Tago K, Mashino T, Kasahara T. Anti-inflammatory activity of structurally related flavonoids, Apigenin, Luteolin and Fisetin. Int Immunopharmacol 2011; 11(9): $1150-9$

[29] Zhao F, Wang L, Liu K. In vitro anti-inflammatory effects of arctigenin, a lignan from Arctium lappa L., through inhibition on iNOS pathway. J Ethnopharmacol 2009; 122(3): 457-62.

[30] Hyam SR, Lee IA, Gu W, et al. Arctigenin ameliorates inflammation in vitro and in vivo by inhibiting the PI3K/AKT pathway and polarizing M1 macrophages to M2-like macrophages. Eur J Pharmacol 2013; 708(1-3): 21-9.

[31] Choa MK, Janga YP, Kima YC, Kim SG. Arctigenin, a phenylpropanoid dibenzylbutyrolactone lignan, inhibits MAP kinases and AP-1 activation via potent MKK inhibition: the role in TNF- $\alpha$ inhibition. Int Immunopharmacol 2004; 4(10-11): 1419-29.

[32] Kou X, Qi S, Dai W, Luo L, Yin Z. Arctigenin inhibits lipopolysaccharide-induced iNOS expression in RAW264.7 cells through suppressing JAK-STAT signal pathway. Int Immunopharmacol 2011; 11(8): 1095-102.

[33] Predes FS, Carvalho JE, Foglio MA, Dolder H, Ruiz AL Antioxidative and in vitro antiproliferative activity of Arctium lappa root extracts. BMC Complement Altern Med 2011; 11(25): 11-25.

[34] Funk JL, Oyarzo JN, Frye JB, et al. Turmeric extracts containing curcuminoids prevent experimental rheumatoid arthritis. J Nat Prod 2006; 69(3): 351-5.

[35] Chandran B, Goel A. A randomized, pilot study to assess the efficacy and safety of curcumin in patients with active rheumatoid arthritis. Phytother Res 2012; 26(11): 1719-25.

[36] Zhou H, Beevers CS, Huang S. The targets of curcumin. Curr Drug Targets 2011; 12: 332-47.

[37] Strimpakos AS, Sharma RA. Curcumin: Preventative and therapeutic properties in laboratory studies and clinical trials. Antioxid Redox Sign 2008; 10: 511-45.

[38] Roshak AK, Callahan JF, Blake SM. Small-molecule inhibitors of $\mathrm{NF}-\mathrm{kB}$ for the treatment of inflammatory joint disease. Curr Opin Pharmacol 2002; 2(3): 316-21

[39] Basnet P, Skalko-Basnet N. Curcumin: an anti-Inflammatory Molecule from a Curry Spice on the Path to Cancer Treatment. Molecules 2011; 16(6): 4567-98.

[40] Fujisawa S, Atsumi T, Ishihara M, Kadoma Y. Cytotoxicity, ROSgeneration activity and radical-scavenging activity of curcumin and related compounds. Anticancer Res 2004; 24(2B): 563-69.

[41] Haqqi TM, Anthony DD, Gupta S, et al. Prevention of collageninduced arthritis in mice by a polyphenolic fraction from green tea. Proc Natl Acad Sci USA 1999; 96(8): 4524-29.

[42] Ahmed S, Rahman A, Hasnain A, Lalonde M, Goldberg VM, Haqqi TM. Green tea polyphenol epigallocatechin-3-gallate 
inhibits the IL-1 beta-induced activity and expression of cyclooxygenase-2 and nitric oxide synthase-2 in human chondrocytes. Free Radic Biol Med 2002; 33(8): 1097-105.

[43] Singh R, Ahmed S, Islam N, Goldberg VM, Haqqi TM. Epigallocatechin-3-gallate inhibits interleukin-1 $\beta$-induced expression of nitric oxide synthase and production of nitric oxide in human chondrocytes: suppression of nuclear factor $\mathrm{\kappa B}$ activation by degradation of the inhibitor of nuclear factor kappaB. Arthritis Rheum 2002; 46(8): 2079-86.

[44] Lin SK, Chang HH, Chen YJ, et al. Epigallocatechin-3-gallate diminishes CCL2 expression in human osteoblastic cells via upregulation of phosphatidylinositol 3-Kinase/Akt/ Raf-1 interaction: a potential therapeutic benefit for arthritis. Arthritis Rheum 2008; 58(10): 3145-56.

[45] Singh R, Akhtar N, Haqqi TM. Green tea polyphenol epigallocatechin-3-gallate: inflammation and arthritis. Life Sci 2010; 86(25-26): 907-18.

[46] Ahmed S. Green tea polyphenol epigallocatechin 3-gallate in arthritis: progress and promise. Arthritis Res Ther 2010; 12: 208.

[47] Nanjo F, Mori M, Goto K, Hara Y. Radical Scavenging Activity of Tea Catechins and Their Related Compounds. Biosci Biotechnol Biochem 1999; 63(9): 1621-3.

[48] Kurien BT, Hensley K, Bachmann M, Scofield RH. Oxidatively modified autoantigens in autoimmune diseases. Free Radic Biol Med 2006; 41(4): 549-56.

[49] Meng Q, Velalar CN, Ruan R. Effects of epigallocatechin-3-gallate on mitochondrial integrity and antioxidative enzyme activity in the aging process of human fibroblast. Free Radic Biol Med 2008; 44(6): 1032-41.

[50] Hirata A, Murakami Y, Atsumi T, et al. Ferulic Acid Dimer Inhibits Lipopolysaccharide-stimulated Cyclooxygenase-2 Expression in Macrophages. In Vivo 2005; 19(5): 849-53.

[51] Qin J, Shang L, Ping A, et al. TNF/TNFR signal transduction pathway-mediated anti-apoptosis and anti-inflammatory effects of sodium ferulate on IL-1 $\beta$-induced rat osteoarthritis chondrocytes in vivo. Arthritis Res Ther 2013; 15(3): 407.

[52] Dong W, Liu S, Yu B, Wu D, Luo H, Yu J. Ameliorative effects of sodium ferulate on experimental colitis and their mechanisms in rats. World J Gastroenterol 2003; 9(11): 2533-8.

[53] Shang L, Qin J, Chen LB, Liu BX, Jacques M, Wang H. Effects of sodium ferulate on human osteoarthritic chondrocytes and osteoarthritis in rats. Clin Exp Pharmacol Physiol 2009; 36(9): 9128.

[54] Roy S, Metya SK, Sannigrahi S, Rahaman N, Ahmed F. Treatment with ferulic acid to rats with streptozotocin-induced diabetes: effects on oxidative stress, pro-inflammatory cytokines, and apoptosis in the pancreatic $\beta$ cell. Endocrine 2013; 44(2): 369-79.

[55] Chung, Soo-Jin, Kim, Tae-Yeon, et al. Gallic Acid Diminishes Cellular Proliferation and Pro-Inflammatory Gene Expressions in Fibroblast Like Synoviocytes from Patients with Rheumatoid Arthritis. Arthritis Rheum 2010; 62(10): 27.

[56] Yoon CH, Chung SJ, Lee SW, Park YB, Lee SK, Park MC. Gallic acid, a natural polyphenolic acid, induces apoptosis and inhibits proinflammatory gene expressions in rheumatoid arthritis fibroblast-like synoviocytes. Joint Bone Spine 2013; 80(3):274-9.

[57] Kroes BH, Berg VAJ, Dijk HV, Labadie RP, Quarles HC. Antiinflammatory activity of gallic acid. Planta Med 1992; 58(6): 499504.

[58] Kim S, Jun C, Suk K, et al. Gallic Acid Inhibits Histamine Release and Pro-inflammatory Cytokine Production in Mast Cells. Toxicol Sci 2006; 91(1): 123-31.

[59] Zhang Y, Dong J, Zhang Q, et al. Genistein inhibit cytokines or growth factor-induced proliferation and transformation phenotype in fibroblast-like synoviocytes of rheumatoid arthritis. Inflammation 2012; 35(1): 377-87.

[60] Wang J, Zhang Q, Jin S, He D, Zhao S, Liu S. Genistein modulate immune responses in collagen-induced rheumatoid arthritis model. Maturitas 2008; 59(4): 405-12
[61] Ji G, Zhang Y, Yang Q, et al. Genistein suppresses LPS-induced inflammatory response through inhibiting NF- $\mathrm{KB}$ following AMP kinase activation in RAW 264.7 macrophages. PloS One 2012; 7(12): e53101.

[62] Hämäläinen M, Nieminen R, Vuorela P, Heinonen M, Moilanen E. Anti-inflammatory effects of flavonoids: genistein, kaempferol, quercetin, and daidzein inhibit STAT-1 and NF-kappaB activations, whereas flavone, isorhamnetin, naringenin, and pelargonidin inhibit only NF-kappaB activation along with their inhibitory effect on iNOS expression and NO production in activated macrophages. Mediators Inflamm 2007: 45673.

[63] Jia Z, Babu PV, Si H, et al. Genistein inhibits TNF- $\alpha$-induced endothelial inflammation through the protein kinase pathway A and improves vascular inflammation in C57BL/6 mice. Int J Cardiol 2013; 168(3): 2637-45.

[64] Yoon HY, Lee EG, Lee $\mathrm{H}$, et al. Kaempferol inhibits IL-1ßinduced proliferation of rheumatoid arthritis synovial fibroblasts and the production of COX-2, PGE2 and MMPs. Int J Mol Med 2013; 32(4): 971-7.

[65] Gong J, Shin D, Han S, Kim J, et al. Kaempferol suppresses eosionphil infiltration and airway inflammation in airway epithelial cells and in mice with allergic asthma. J Nutr 2012; 142(1): 47-56.

[66] Xiao P, Hao Y, Zhu X, Wu X. p53 contributes to quercetin-induced apoptosis in human rheumatoid arthritis fibroblast-like synoviocytes. Inflammation 2013; 36(2): 272-8.

[67] Kaussa T, Ramberta J, Fawazb F, et al. Therapeutic and preventive properties of quercetin in experimental arthritis correlate with decreased macrophage inflammatory mediators. Biochem Pharmacol 2006; 72(10): 1304-10.

[68] Choi EJ, Bae SC, Yu R, Youn J, Sung MK. Dietary vitamin E and quercetin modulate inflammatory responses of collagen-induced arthritis in mice. J Med Food 2009; 12(4): 770-5.

[69] Jackson JK, Higo T, Hunter WL, Burt HM. The antioxidants curcumin and quercetin inhibit inflammatory processes associated with arthritis. Inflamm Res 2006; 55(4): 168-75.

[70] Nishizaki T, Kanno T. Resveratrol: a Candidate Drug for Treating Rheumatoid Arthritis. 1st ed. In: Lemmey A, Ed. Rheumatoid Arthritis - Treatment. Shanghai, In Tech china 2012: pp. 270-84.

[71] Byun HS, Song JK, Kim YR, et al. Caspase-8 has an essential role in resveratrol-induced apoptosis of rheumatoid fibroblast-like synoviocytes. Rheumatology 2008; 47(3): 301-8.

[72] Shakibaei M, Csaki C, Nebrich S, Mobasheri A. Resveratrol suppresses interleukin-1beta-induced inflammatory signaling and apoptosis in human articular chondrocytes: potential for use as a novel nutraceutical for the treatment of osteoarthritis. Biochem Pharmacol 2008; 76(11): 1426-39.

[73] Liu F, Hung L, Wu W, et al. Chondroprotective effects and mechanisms of resveratrol in advanced glycation end productsstimulated chondrocytes. Arthritis Res Ther 2010; 12(5): R167.

[74] Xuzhu G, Leung BP, Howe HS, et al. Resveratrol modulates murine collagen-induced arthritis by inhibiting Th17 and B-cell function. Ann Rheum Dis 2012; 71(1): 129-35.

[75] Ates O, Cayli S, Altinoz E, et al. Neuroprotection by resveratrol against traumatic brain injury in rats. Mol Cell Biochem 2007; 294(1-2): 137-44.

[76] Leonarda SS, Xiab C, Jiangb B, et al. Resveratrol scavenges reactive oxygen species and effects radical-induced cellular responses. Biochem Biophys Res Commun 2003; 309(4): 1017-26.

[77] Dave M, Attur M, Palme G, et al. The antioxidant resveratrol protects against chondrocyte apoptosis via effects on mitochondrial polarization and ATP production. Arthritis Rheum 2008; 58(9): 2786-97.

[78] Csaki C, Keshishzadeh N, Fischer K, Shakibaei M. Regulation of inflammation signalling by resveratrol in human chondrocytes in vitro. Biochem Pharmacol 2008; 75(3): 677-87. 